\title{
Killing of S. mutans Bacteria Using a Plasma Needle at Atmospheric Pressure
}

\author{
J. Goree, Member, IEEE, Bin Liu, David Drake, and Eva Stoffels
}

\begin{abstract}
Streptococcus mutans (S. mutans) bacteria were killed using a low-power millimeter-size atmospheric-pressure glowdischarge plasma or plasma needle. The plasma was applied to a culture of $S$. mutans that was plated onto the surface of an agar nutrient in a Petri dish. $S$. mutans is the most important microorganism for causing dental caries. A spatially resolved biological diagnostic of the plasma is introduced, where the spatial pattern of bacterial colonies in the sample was imaged after plasma treatment and incubation. For low-power conditions that would be attractive for dentistry, images from this biological diagnostic reveal that $S$. mutans was killed within a solid circle with a 5-mm diameter, demonstrating that site-specific treatment is possible. For other conditions, which are of interest for understanding plasma transport, images show that bacteria were killed with a ringshaped spatial pattern. This ring pattern coincides with a similar ring in the spatial distribution of energetic electrons, as revealed by Abel-inverted images of the glow. The presence of the radicals $O H$ and $O$ was verified using optical-emission spectroscopy.
\end{abstract}

Index Terms-Atmospheric glow discharge, disinfection, microorganisms, microplasma, nonthermal plasma, plasma applications, sterilization.

\section{INTRODUCTION}

A TMOSPHERIC-PRESSURE glow discharges show a considerable promise for decontamination and disinfection applications, where surfaces are exposed to plasma in order to destroy microorganisms. Of interest here are nonthermal glow discharges, i.e., nonequilibrium plasmas with gas at nearly the same temperature as the ambient gas, in contrast to thermal plasmas or arcs [1]-[7]. For biomedical applications, atmospheric conditions are essential because samples cannot be inserted into a vacuum chamber.

Atmospheric glow discharges can produce short-lived chemical species, which are propelled toward a surface that is to be treated. The short life of these species is desirable because they do not remain after the treatment is completed.

With appropriately designed electrodes, power supply, and gas supply, an atmospheric-pressure glow discharge can be operated stably, below the glow-to-arc transition. This generally requires one or more of the following design features: using inert gas such as helium to lower the breakdown voltage, a significant gas flow rate, high-frequency power, a cathode with

Manuscript received December 6, 2005; revised March 31, 2006.

J. Goree and B. Liu are with the Department of Physics and Astronomy, University of Iowa, Iowa City, IA 52242 USA (e-mail:

D. Drake is with Dows Institute for Dental Research, Department of Endodontics, College of Dentistry, University of Iowa, Iowa City, IA 52242 USA.

E. Stoffels is with the Faculty of Biomedical Engineering, Eindhoven University of Technology, 5600 MB Eindhoven, The Netherlands.

Digital Object Identifier 10.1109/TPS.2006.878431 sharp features to increase the local electric field, and a dielectric barrier on the electrodes. The device studied here has most of these features.

Our "plasma needle" device produces a small-diameter lowpower atmospheric-pressure glow discharge. It is intended for dental or medical applications [8]-[15]. Radio-frequency high voltage is applied to a single needle electrode located inside a concentric gas-flow nozzle. The nozzle has a diameter of a few millimeters, and the plasma that flows out of the nozzle has a comparable diameter. This design is similar to the socalled microbeam plasma generator [7]. The nozzle is placed a few millimeters from the surface that is to be treated, and the plasma jet is directed onto that surface. When desired, the plasma needle can be operated at a power so low that the glow is barely visible to the unaided eye.

Using small-diameter plasma allows site-specific disinfection of spots with a diameter of a few millimeters. This small size distinguishes the plasma needle from larger atmosphericglow devices intended to sterilize large-area contaminated surfaces [16]-[20]. For dental or medical use, a disinfection method should be precise enough to treat small areas.

One proposed application for the plasma needle is the treatment of dental caries [11]. The most significant cariogenic microorganism is Streptococcus mutans (S. mutans). This is a gram-positive, facultatively anaerobic microorganism that form biofilms on teeth (dental plaque), penetrates into fissures, and erodes the enamel and dentine. Since it is a facultative anaerobe, it can grow in the presence of oxygen, but it prefers oxygen-poor conditions. It penetrates into fissures where it is well protected from oxygen and from the natural antimicrobial activity of saliva. Its temperature range for growth is $30^{\circ} \mathrm{C}$ to $47^{\circ} \mathrm{C}$, with optimal growth at human body temperature, $37^{\circ} \mathrm{C}$. It is inactivated at temperatures above $60{ }^{\circ} \mathrm{C}$ [21].

Individual $S$. mutans bacteria are relatively easy to kill. However, they form thick biofilms in the presence of a sucrose consisting of high-molecular weight glucans. This layer is rather difficult to penetrate. Antibacterial treatment methods that have been used for $S$. mutans include rinsing with chemical solutions such as chlorhexidine [22], [23] and laser irradiation [24].

In clinical practice, caries are treated by drilling, which removes healthy tissue along with the infected dentine. It would be desirable to ensure disinfection before placement of restorative materials by destroying the bacteria in a cavity without harming healthy tissue. Unfortunately, current chemotherapeutic agents, such as chlorhexidine, have undesirable side effects, including disagreeable taste and stains [22]. Plasma needle treatment is unlikely to have these disadvantages, and it offers some promise for painless treatment as well. 
The plasma needle produces short-lived chemical species in the gas phase. These can impinge on a tooth's surface, and they can dissolve into a liquid [15]. Unlike liquid rinses with bactericidal ingredients that linger in the mouth after treatment, the plasma needle produces bactericidal agents locally. Once the treatment is completed, no excess radicals remain, due to recombination among themselves or by reacting with ambient air and water molecules. Thus, the bactericidal agents have a naturally short lifetime, which is an attractive feature.

At the present time, it is not known exactly which species produced by a plasma needle applies the greatest bactericidal effect, or where it is formed. Radical formation can occur either in the gas phase or in a liquid. In both cases, the process begins with energetic electrons in the plasma. In the gas phase, atomic oxygen $\mathrm{O}$ and hydroxyl $\mathrm{OH}$ radicals are produced by dissociation of air molecules $\mathrm{H}_{2} \mathrm{O}$ and $\mathrm{O}_{2}$ as a result of electron impact [12], [15]. These two radicals, $\mathrm{O}$ and $\mathrm{OH}$, are both known to have a bactericidal effect [26], [27].

We also suggest that radical formation in the liquid phase is possible. This process would begin with helium atoms in the gas phase being excited to a metastable state due to electron impact. These metastable atoms subsequently enter the aqueous sample, where they produce radicals in situ by dissociating $\mathrm{H}_{2} \mathrm{O}$. This process can occur only if metastable helium atoms are not quenched by collisions in the atmosphere before contacting the aqueous sample.

The surface temperature of a tooth during treatment is therapeutically not important. It was previously demonstrated [11] that the plasma needle can easily be operated at a power low enough that the temperature of a tooth's pulp will not be elevated by more than $5.5{ }^{\circ} \mathrm{C}$, a level at which pulpal necrosis can occur [25]. Unlike the pulp, the tooth's surface is unharmed by extended exposure to much higher temperatures, as for example when consuming hot coffee. Dental tissue has a low thermal conductivity, so that superficial heating of a tooth does not necessarily heat the pulp significantly [11].

In this paper, we come to three conclusions. First, for operating conditions attractive for clinical dentistry, plasma needle treatment:

1) can kill the most important cariogenic bacterium S. mutans;

2) requires a treatment time of tens of seconds;

3) has a reproducible killing effect;

4) is site specific with a treatment spot that is a solid circle several millimeters in diameter.

Second, at other operating conditions, attained at higher voltages or smaller needle-to-sample separations than would be attractive for clinical treatment, results are reported that are interesting for gaining insight into plasma transport processes. The shape of the spot where $S$. mutans are killed depends on the plasma operating parameters: instead of a solid circle, it can also be a ring. An understanding of the ring shape can be gained by imaging the glow. Third, we introduce a biological diagnostic of plasmas. This method provides a sensitive and spatially resolved image of a cross section of an impinging plasma. It is demonstrated here by imaging the spatial pattern of bacterial killing in site-specific plasma treatment.

\section{PROCEDURE}

\section{A. Sample Preparation}

Bacteria culture was plated onto agar, forming a bacterial lawn. Plastic Petri dishes were filled to a depth of $4 \mathrm{~mm}$ with agar. Agar is an aqueous growth medium with a jellylike consistency; it has a brown color due to its high-protein nutrient components. After cooling and solidification, dishes were inoculated with $S$. mutans using a spiral-plating technique. This was done in a Spiral Biotech Autoplate 4000, which resembles a phonograph record player, with a turntable that rotates the dish while a dispensing stylus applies the bacterial suspension. The bacterial concentration was $10^{6}-10^{7}$ colony-forming units (CFUs) per milliliter suspension. The resulting spiral-shaped line formed a bacterial lawn covering the surface of the agar. A 12-mm-diameter circle in the center of the dish was not inoculated.

Spiral-plating yields a sample with bacteria localized on a surface, and not dispersed in a volume. This is suitable for testing the plasma needle, because plasma treatment is essentially a surface treatment. However, it has also been shown that plasmagenerated radicals can be absorbed into the volume of a water sample, with a penetration depth of several millimeters [15].

\section{B. Plasma Needle Apparatus}

We used a plasma needle similar to the design reported in [11]. It consists of a handpiece, a gas supply, and a highfrequency generator. The handpiece [Fig. 1(a)] has three fittings: a gas inlet, an electrical feedthrough and a nozzle. The most important features are inside the nozzle [Fig. 1(b)]. At the center of the nozzle is a tungsten neurology needle, with a diameter of $0.2 \mathrm{~mm}$ and a pencil-shaped taper of length $0.6 \mathrm{~mm}$. The tip was sharp, but it dulled after operating the plasma over time, as indicated by a slight increase in breakdown voltage. The needle was concentric with a cylindrical glasstube nozzle, which has an inside diameter $D=4 \mathrm{~mm}$ and an outside diameter of $6.35 \mathrm{~mm}$. The needle shaft was covered with a ceramic insulator tube, leaving a length $L=5.7 \mathrm{~mm}$ of the needle exposed to gas. Along this length, and at the tip itself, a glow can form. The nozzle was flush with the needle tip.

We used pure helium, which was found in earlier tests to offer a lower breakdown voltage and more stability from the glowto-arc transition, as compared to other gas mixtures [11]. It is unattractive to add gases such as $\mathrm{O}_{2}$ directly into the gas feed because this diminishes the production of radicals, presumably due to electron attachment inside the nozzle [15]. For producing radicals in the gas phase, we rely on mixing air into the flow downstream of the nozzle.

The needle electrode was powered by a $7.17-\mathrm{MHz}$ radiofrequency high voltage. To provide a return current, a grounded metal plate was positioned below the plastic Petri dish, as shown in the scale drawing of Fig. 1(b) and the sketch of Fig. 1(c).

Clinical applications would require a compact portable generator, which should couple radio-frequency power efficiently to the plasma so that excessive voltages are not required to achieve breakdown. The generator setup we used, however, 


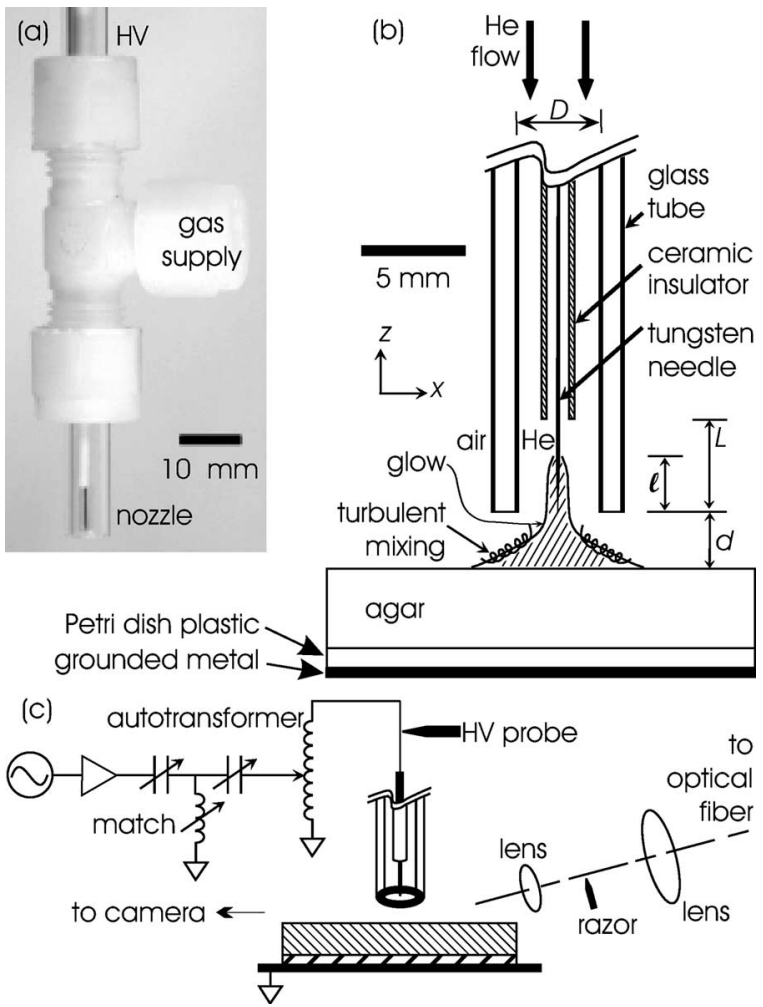

Fig. 1. Plasma needle setup. (a) Handpiece was a nylon Swagelok tee with the nozzle pointed downward. (b) This scale drawing of the nozzle includes a sketch showing where the glow was visible. Bacteria were plated on the surface of the agar. (c) RF network and optical glow diagnostics, not drawn to scale.

did not match efficiently to the high impedance of the plasma. Most of the radio-frequency power was deposited into cables or otherwise in the network, and not in the plasma. Moreover, our needle and nozzle design is probably not optimal. As a result, attaining gas breakdown required a higher voltage of typically $600 \mathrm{~V}$ peak to peak, as compared to $200 \mathrm{~V}$ for another plasma needle setup [11].

Our network consisted of a tee-configuration matchbox intended for amateur radio, followed by an autotransformer coil to boost the output impedance, as sketched in Fig. 1(c). The peak-to-peak voltage was measured with a digital-storage oscilloscope using a Tektronix P5100 high-voltage probe that contacted the RF conductor. The probe location was $80 \mathrm{~cm}$ from the needle tip; therefore, the voltage measured does not necessarily indicate the voltage on the tip itself. Thus, there are two reasons our voltage measurements are useful only for relative comparisons, and not for comparison to other setups: our network did not match the plasma impedance, and our probe did not necessarily measure the voltage at the needle's tip.

The gas flow from the nozzle is important for several reasons. First, it is helpful in achieving breakdown. Second, the flow mixes downstream from the nozzle with atmospheric gases including $\mathrm{N}_{2}, \mathrm{O}_{2}$, and $\mathrm{H}_{2} \mathrm{O}$. Third, the gas flow propels radicals and metastable toward the surface to be treated. Fourth, the flow diameter helps determine the diameter of the treated spot on the sample. The flow rate was an adjustable parameter, and it was measured using a Sierra mass flow meter. The flow velocity $V$ in the glass-tube nozzle is proportional to the flow rate; at a flow rate of 1 standard liters per minute (SLPM), the velocity is $V=1.3 \mathrm{~m} / \mathrm{s}$. This gas flow is comfortable in the mouth.

We positioned the handpiece using a mechanical setup with the needle pointed downward toward the sample. The sample's agar surface was horizontal, so that the gas flow impinged the sample's surface at $90^{\circ}$ incidence. The handpiece was held in a mount that allowed raising and lowering it.

\section{Optical Diagnostics for the Glow}

We tested for the presence of radicals using optical-emission spectroscopy (OES). At one end of an optical fiber, light was coupled into the entrance slit of an Ocean Optics HR2000 spectrometer, with a $300-$ lines $/ \mathrm{mm}$ grating. The other end of the fiber collected light focused by a pair of fused silica convex lenses [Fig. 1(c)]. To detect only light produced downstream from the nozzle, we positioned a razor blade in the focal plane between the lenses to block light produced inside the glass tube.

We also imaged the glow. This was done under the same operating conditions as for a bactericidal treatment, but with the plasma impinging on a clean 5-mm-thick glass plate substituted for the Petri dish with agar. The Dalsa 1M30 camera that was used has a 12-bit monochrome charge-coupled device (CCD) with a linear response so that the pixel values recorded are proportional to the actual light intensity. The exposure time was $1 / 30 \mathrm{~s}$; longer exposures are impractical because small atmospheric winds deflect the glow. Using a $105-\mathrm{mm}$ focallength Nikon microlens with no spectral filter, we imaged light in the visible and near IR, which consisted mostly of $\mathrm{He}$ spectral lines. When viewing these images of the glow, it is useful to remember that brightness in the image reveals only the presence of energetic electrons, and not necessarily the presence of bactericidal agents. Energetic electrons can only produce radicals if air is mixed into the flow.

To reveal the true spatial profile of the emission, we transformed the images using Abel inversion. The observed image $i(x, z)$ is a projection of the emission onto an $x z$ plane, where $z$ is the height above the sample surface. To compute the emission function $I(r, z)$, we used the reverse Abel transform

$$
I(r, z)=-\frac{1}{\pi} \int_{r}^{\infty} \frac{d i(x, z)}{d x} \frac{d x}{\sqrt{x^{2}-r^{2}}}
$$

which assumes a circularly symmetric plasma cross section.

\section{Plasma Treatment}

There were four adjustable parameters for our plasma treatment. The exposure time was varied in a range from 10 to $120 \mathrm{sec}$, the needle-to-sample separation $d$ from 2 to $4 \mathrm{~mm}$, the RF peak-to-peak voltage from 600 to $900 \mathrm{~V}$, and the gas flow from 0.2 to 4.0 SLPM.

To begin, we raised the handpiece, installed a Petri dish with its center immediately below the needle, and then lowered the handpiece to the desired separation $d$. At this time, the plasma impinged on the center of the dish, which was not inoculated. 


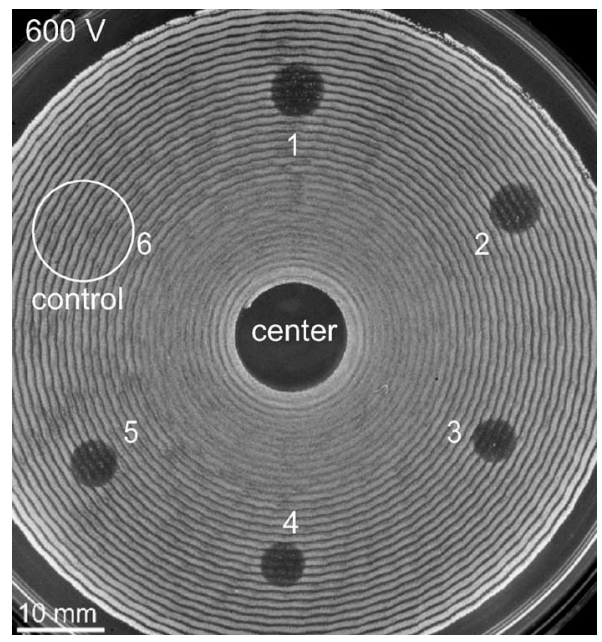

Fig. 2. Petri dish imaged after treatment and incubation. A light color indicates living bacteria colonies. The spiral pattern is due to the inoculation method; the center was never inoculated. Plasma treatment, applied to spots $1-5$, killed the $S$. mutans bacteria, as indicated by the dark color. Spot 6 was a control, treated with gas flow but no plasma; it appears the same as the surrounding untreated areas. Treatment conditions were 1.5 SLPM, $d=3 \mathrm{~mm}$, $V_{\mathrm{pp}}=600 \mathrm{~V}$, and exposure time $30 \mathrm{~s}$.

Next, we began plasma treatment by positioning the Petri dish so that the plasma impinged on a desired spot in the inoculated portion of the dish. At the end of the exposure time, we moved the dish to treat another spot. We repeated this step to treat a total of five spots on the dish, as shown in Fig. 2.

Finally, as the control for the experiment, we moved the Petri dish to treat a sixth spot, and simultaneously we switched the plasma off while allowing the gas to continue flowing. As a control, this spot indicates the effects of helium flow without any plasma-generated species.

\section{E. Imaging}

In this paper, we introduce a new spatially resolved method of detecting the effects of plasma treatment. From the perspective of a plasma physicist, this method can be used as a biological diagnostic of plasmas.

The method makes use of the fact that bacteria that are not killed by treatment can be incubated to grow a visible biofilm. This biofilm has a whitish color that contrasts with the dark natural color of agar. The dark color of agar is observed in regions where bacteria were killed. The contrast between whitish biofilms of living bacteria and dark regions where bacteria were killed allows high-resolution spatially resolved imaging of treatment spots.

The imaging procedure was as follows. After treatment with the plasma needle, the Petri dishes were covered to prevent atmospheric contamination. At this time, the treated spots were not yet identifiable by any color change in the sample. The dishes were then installed in a $\mathrm{CO}_{2}$ incubator at $37{ }^{\circ} \mathrm{C}$ for $48 \mathrm{~h}$ so that bacteria multiplied and formed colonies. After incubation, we imaged the dishes with a digital color camera using white photographer's lights for illumination. The image resolution was $48 \mu \mathrm{m}$ per pixel.

An image of an agar plate after incubation is shown in Fig. 2. Features to note include a light color indicating a biofilm of

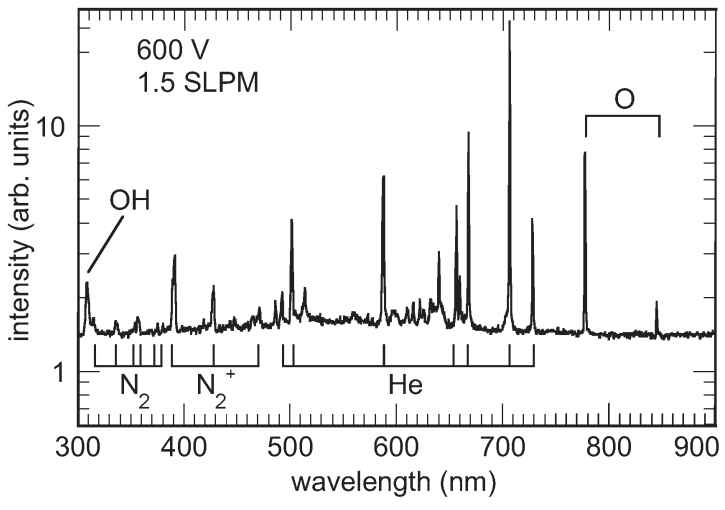

Fig. 3. Spectrum of the optical emission from the glow, downstream of the nozzle, for the same conditions as Fig. 2. Note the lines for $\mathrm{OH}$ and $\mathrm{O}$, which are bactericidal agents produced by the plasma. Wavelengths are listed in Table I.

living bacterial colonies, and the dark color of agar without living colonies. Dark spots can be identified where bacteria were killed during plasma treatment. The large central spot on the dish was never inoculated, so that it also has the dark color of the agar.

Thus, we have a spatially resolved diagnostic that allows us to view the site-specific treatment. Color is our primary indicator of bactericidal effect. For our present purpose of demonstrating site-specific killing, it is enough to reveal whether bacteria were killed and where.

In the future, this diagnostic could be developed further to provide quantitative measurements of CFUs. This could be done by calibrating the images of the biofilm, using an additional quantitative method. In previous experiments with E. coli, the fraction of CFU surviving treatment was quantified [11], but without the spatial resolution of our current method.

\section{RESULTS}

\section{A. Optical-Emission Spectra Results}

The optical-emission spectrum verifies the presence of $\mathrm{O}$ and $\mathrm{OH}$ radicals. This is shown in Fig. 3 and Table I. Similar spectra for a plasma needle device were reported previously [11]. The radicals $\mathrm{O}$ and $\mathrm{OH}$ are known to be bactericidal.

\section{B. Bacteriology Results}

1) Conditions Desirable for Dental Treatment: The killing of $S$. mutans by plasma treatment is shown by the dark treatment spots in the Petri dishes, after incubation. In Fig. 2, spots 1-5 were treated identically and their darkness indicates a bactericidal effect. This effect shows consistent reproducibility in all five treated spots.

Control spot 6 , on the other hand, appears the same as its surroundings. This control spot was treated with the handpiece positioned at the same distance $d$, the same gas flow, and the same duration as for spots $1-5$, but the high voltage that sustains the plasma was turned off.

Comparing spots 1-5 with the control spot 6 allows us to conclude that the bactericidal effect is due to the plasma. However, it remains unknown which plasma-generated species 
TABLE I

For the SPECTRAL Lines OBSERVED In Fig. 3, STANDARD WaVELEnGths ARe Listed Here [28], [29]

\begin{tabular}{llll}
\hline \hline Species & Wavelength $(\mathrm{nm})$ & Species & $\begin{array}{c}\text { Wavelength } \\
(\mathrm{nm})\end{array}$ \\
\hline $\mathrm{O}$ & 777.19 & $\mathrm{~N}_{2}{ }^{+}$ & 391.44 \\
& 844.64 & & 427.81 \\
& & 470.92 \\
$\mathrm{OH}$ & 306.4 system: & & \\
& 306.36 & & \\
& 306.72 & & \\
& 307.8 & & 315.93 \\
& 308.9 & & 337.13 \\
$\mathrm{He}$ & 492.19 & $\mathrm{~N}_{2}$ & 353.67 \\
& 501.57 & & 357.69 \\
& 587.56 & 375.54 \\
& 656.01 & 380.49 \\
& 667.82 & & \\
\hline \hline
\end{tabular}

is most responsible for the killing. As discussed in Section I, candidates include $\mathrm{O}$ and $\mathrm{OH}$ radicals produced by electronimpact dissociation in the gas or possibly metastable He produced in the gas that subsequently enters the aqueous sample and produces radicals in situ by dissociating $\mathrm{H}_{2} \mathrm{O}$.

The shape of the treatment spot achieved in Fig. 2 indicates that the plasma-needle can provide a desirable site-specific treatment. For clinical applications in dentistry, site-specific treatment with a diameter of a few millimeters is required and Fig. 2 demonstrates that this has been achieved. The treatment spot in Fig. 2 is a solid circle of diameter $5 \mathrm{~mm}$. Our imaging method was essential for measuring the size and shape of this site-specific treatment spot.

Conditions leading to a homogenous circle are, in general, low voltage, moderate gas flow rate, large separation $d$, and a moderately short exposure time. For Fig. 2, the parameters were $V_{\mathrm{pp}}=600 \mathrm{~V}, 1.5 \mathrm{SLPM}, d=3 \mathrm{~mm}$, and exposure time $30 \mathrm{~s}$.

The surface temperature during this treatment did not exceed $40{ }^{\circ} \mathrm{C}$, so that the bactericidal effect can be attributed to chemical rather than thermal means. We measured the surface temperature in a test by applying the plasma directly to liquidcrystal strips that were positioned on top of glass plates of the same thickness as the agar and Petri dish. Operating at the same conditions as for Fig. 2, the temperature exceeded $35^{\circ} \mathrm{C}$ after $12 \mathrm{~s}$ or more exposure time, and it never exceeded $40^{\circ} \mathrm{C}$ within $30 \mathrm{~s}$ of exposure. We verified these results in separate tests using an irreversible temperature-indicator strip. We expect that the surface temperature during treatment of the agar was even lower, because agar is aqueous and can evaporate, thereby cooling the sample. Therefore, this method yields an upper limit for the actual surface temperature during treatment.

Another scheme that we found useful for measuring temperature was embedding the temperature-sensitive strips inside the agar, at a 2-mm depth; this scheme measures actual temperature during treatment, including the effects of evaporation; this (a)
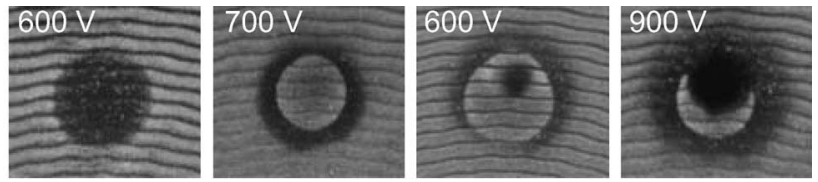

(b)
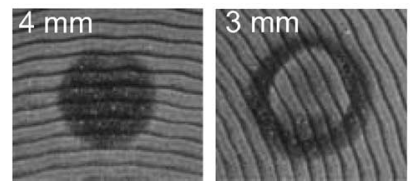

Fig. 4. Treatment spots imaged after treatment and incubation, for various plasma treatment parameters. In each row, one parameter was adjusted. Columns are aligned to show similar killing patterns: a solid circle in the left column, a ring in the second column, and a ring with a central spot in the two columns to the right. The latter two columns correspond to conditions near the glow-to-arc transition. (a) $V_{\mathrm{pp}}$ was increased. (b) Separation $d$ was decreased. Except where indicated otherwise, parameters were $1.5 \mathrm{SLPM}, d=3 \mathrm{~mm}$, $V_{\mathrm{pp}}=800 \mathrm{~V}$, and exposure time $30 \mathrm{~s}$.

scheme yields a lower limit for the surface temperature, because of the thermal conductivity and heat capacity of the agar. For the conditions here that led to a homogeneous circle, both methods (temperature sensitive strips embedded in agar or applied to the surface of glass plates) indicated a temperature below $40{ }^{\circ} \mathrm{C}$.

Recalling that $S$. mutans is thermally inactivated only above $60{ }^{\circ} \mathrm{C}$, we conclude that the bactericidal effect observed in Fig. 2 was achieved by chemical rather than thermal means.

2) Other Conditions: In addition to the conditions described above, which are of interest for clinical application, we also found other conditions of interest for another purpose: gaining an understanding the physical processes in the plasma. We summarize these results, for conditions that are not clinically useful (below).

The shape of the treatment spot changed from a homogenous circle to a ring as the voltage was increased or as the separation was decreased. This is seen in Fig. 4(a), where $V_{\mathrm{pp}}$ was increased holding other parameters constant, and in Fig. 4(b), where the separation was decreased while holding other parameters constant.

As the voltage is increased in Fig. 4(a), we see that beginning with the same homogenous spot as in Fig. 2 at low voltage, the spot shape changes to a ring in the second column. There was no significant killing in the ring's center. As the voltage is increased further, nearly to the point of a glow-to-arc transition, a central spot appears inside the ring.

As the separation is decreased, the same trend occurs as when voltage is increased. In Fig. 4(b), the solid circle becomes a ring. We found that the shape of the treatment spot was sensitive to a $0.5 \mathrm{~mm}$ change in the separation, which suggests that for clinical practice it would be necessary to design the handpiece with a mechanical spacer so that the needle tip is reliably positioned at the desired distance.

Unlike the low-power conditions of interest for dental applications, the conditions that lead to ring-shaped killing spot also resulted in an observable level of evaporation. This was observed by an indentation in the agar surface that developed during treatment. The shape of the depression was deepest in the center, so that it did not resemble the shape of the killing spot. We note that our observation that living bacteria were found in the centers of the rings where the evaporation was 

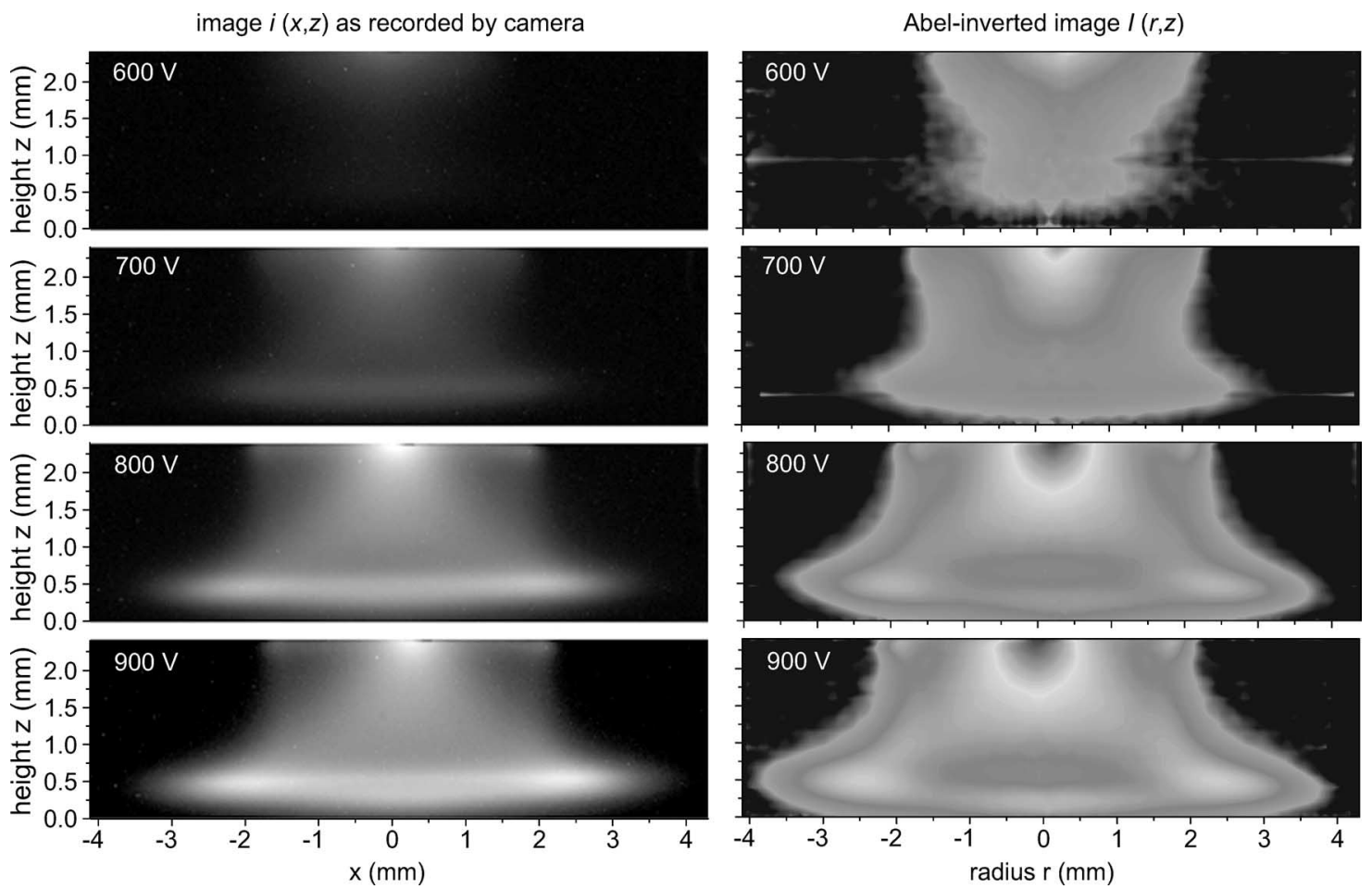

Fig. 5. Images of the glow as viewed from the side. The sample surface at the bottom, $z=0$, was a glass plate substituted for the agar and Petri dish. The nozzle end at $z=3 \mathrm{~mm}$ is not visible in these images. The left column shows images as recorded by the camera, while the right column shows the corresponding images after Abel inversion, using (1), to reveal the radial distribution of the intensity. Parameters were 1.5 SLPM and $d=3 \mathrm{~mm}$.

greatest indicates that only water, and not bacteria, were lost to evaporation. Evaporation provides a cooling effect during treatment.

To summarize, there are three general shapes we observed in the killing pattern: a solid circle, a ring, and a ring with a central spot. The ring shapes are not attractive for clinical treatment, but they do offer a useful indicator for gaining an understanding of physical processes in a plasma. As a step toward gaining that understanding, we will compare our biological diagnostic images to physical images of the glow (next).

\section{Images of the Glow}

Recall that the glow is produced by electron-impact excitation of gas atoms, so that it serves as a visual indicator of the presence of energetic electrons. These energetic electrons are also capable of generating radicals, either in the gas phase by dissociating gas molecules such as $\mathrm{H}_{2} \mathrm{O}$ and $\mathrm{O}_{2}$, or indirectly by generating metastable $\mathrm{He}$ atoms that might possibly enter a liquid and dissociate $\mathrm{H}_{2} \mathrm{O}$ molecules in situ. The light emission is not an indicator of the radicals themselves, but merely an indicator of one of the components required to produce them. To produce $\mathrm{OH}$ or $\mathrm{O}$ radicals in the gas phase requires that $\mathrm{H}_{2} \mathrm{O}$ and $\mathrm{O}_{2}$ be mixed into the glow so that energetic electrons can dissociate them.

Side-view images are shown in Fig. 5. The left column shows the image as recorded by the camera, while the right column shows the Abel-inverted images. The latter are more instructive. For low-power conditions, as in the top row of Fig. 5, the glow is concentrated in a narrow column of diameter $3 \mathrm{~mm}$. For higher power conditions, as in the bottom row of Fig. 5, the glow spreads out to a larger diameter, and it develops into a ring just above the sample surface.

The shape of the glow helps explain the shape of the treatment spots. This is demonstrated in Fig. 6, where the Abelinverted image of the glow is aligned with a corresponding image of the sample. For conditions in the left column, a glow shaped like a narrow column yields a treatment spot that is a solid circle. For conditions in the right column, a glow that is ring-shaped near the sample yields a treatment spot that has a similar ring shape.

This comparison suggests an important role for energetic electrons. Their spatial distribution, as indicated by the glow images, determines the spatial pattern of bactericidal agents that impinge on the sample, as indicated by the biological images. More images and a further discussion of the role of energetic electrons will be presented in a separate publication.

\section{Avoiding the Glow-to-Arc Transition}

At high voltages and small separations $d$, the glow sometimes underwent a transition to a filamentary arc. When this happened, the plasma had a more concentrated shape and there was an audible hissing noise. We found that a reliable visible precursor of nearing the glow-to-arc transition was a large value of the length $l$ of the glow along the needle shaft (Fig. 1). In a stable glow discharge operation, the glow was concentrated only at the tip, $l \ll L$. However, as the voltage was increased or the separation $d$ was decreased, $l$ gradually increased and audible hiss became more noticeable. It is possible that a filament was present within the glow for these higher power conditions, although we have no evidence of this. Finally, the 

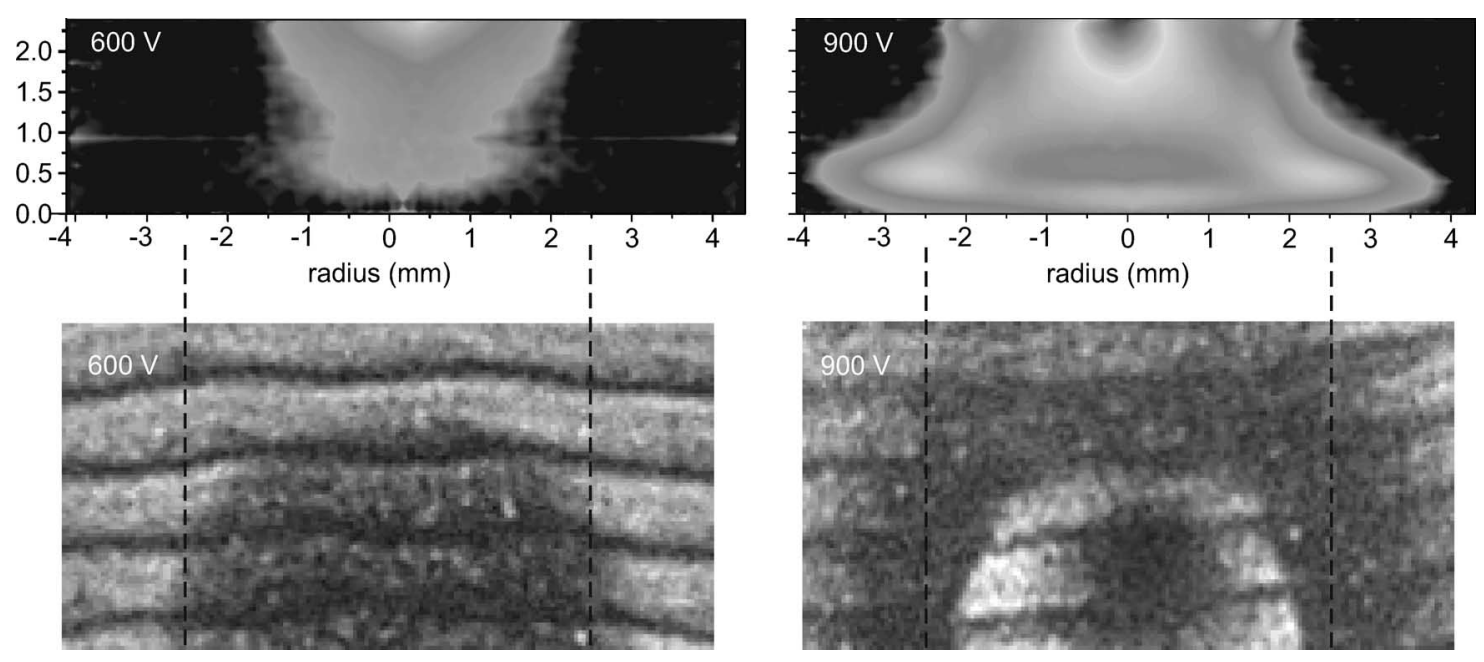

Fig. 6. Abel-inverted images (top) and images of spots on Petri dishes (bottom), at the same scale. For low-power conditions (left column), the glow was a narrow column, yielding a killing pattern with a solid circle of diameter $5 \mathrm{~mm}$. For higher power conditions (right column), the ring, with its 5-mm inside diameter, corresponds to a bright ring in the glow at $(r, z)=(2.5 \mathrm{~mm}, 0.5 \mathrm{~mm})$. Comparing these images of the glow and the Petri dish helps explain the shape of the treatment spot. Parameters were 1.5 SLPM, $d=3 \mathrm{~mm}$, and a 30 -s exposure.

entire glow transitioned to a filamentary arc when the glow touched the insulator $(l=L)$. When this happened, the sample burned where it was touched by the arc, and we discontinued treatment.

For clinical practice, it would be necessary to suppress arcs. We believe that improvements in generator design and nozzle design will make this possible. Otherwise, arcs could occur when the practitioner positions the handpiece too near the surface to be treated.

\section{CONCLUSION}

We demonstrated that the plasma needle is capable of sitespecific killing of bacteria under conditions that are attractive to dentistry. The most important cariogenic bacterium, S. mutans, was killed reproducibly with a treatment time of tens of seconds. This killing was site-specific, occurring within a killing spot that was $5 \mathrm{~mm}$ in diameter.

In addition to operating the plasma needle at conditions that would be attractive for dental clinical treatment, as described above, we also found that it can also be operated under other conditions that are interesting because they provide insight into plasma transport phenomena. Under these conditions of higher voltage or smaller needle-to-sample separation, the treatment results in a ring-shaped killing pattern. Based on Abel-inverted images of the glow, we attribute the ring shape in the killing pattern to a similar ring shape in the spatial distribution of energetic electrons in the plasma.

Imaging of the site-specific treatment was made possible by a biological diagnostic of the plasma that we introduced. This diagnostic yields images showing where microorganisms were killed. We expect that it can find significant future use in gaining an understanding of both the plasma processes and the intended therapeutic application as well. The imaging method could be developed further, to calibrate the color of the biofilm and thereby provide a desirable quantitative measurement of killing.

Transport issues in this paper, including the role of gas flow, diffusion, turbulence, and buoyancy will be reported separately, in another publication. Studies of this type will be useful, among other thing, for providing benchmark data for modeling. We note that modeling efforts for the plasma needle device have recently begun [30].

\section{ACKNOWLEDGMENT}

The authors would like to thank S. Clark, V. Nosenko, F. Skiff, R. Vogel, and J. Wefel for useful discussions.

\section{REFERENCES}

[1] S. Kanazawa, M. Kogoma, T. Moriwaki, and S. Okazaki, "Stable glow plasma at atmospheric pressure," J. Phys. D, Appl. Phys., vol. 21, no. 5, pp. 838-840, May 1988.

[2] S. Okazaki, M. Kogoma, M. Uehara, and Y. Kimura, "Appearance of stable glow discharge in air, argon, oxygen and nitrogen at atmospheric pressure using $50 \mathrm{~Hz}$ source," J. Phys. D, Appl. Phys., vol. 26, no. 5, pp. 889-892, May 1993.

[3] A. Schütze, J. Y. Jeong, S. E. Babayan, J. Park, S. Selwyn, and R. F. Hicks, "The atmospheric-pressure plasma jet: A review and comparison to other plasma sources," IEEE Trans. Plasma Sci., vol. 26, no. 6, pp. 1685-1694, Dec. 1998.

[4] J. Park, I. Henins, H. W. Hermann, and G. S. Selwyn, "An atmosphericpressure plasma source," Appl. Phys. Lett., vol. 76, no. 3, pp. 288-290, Jan. 2000.

[5] — "Discharge phenomena of an atmospheric pressure radio-frequency capacitive plasma source," J. Appl. Phys., vol. 89, no. 1, pp. 20-28, Jan. 2001.

[6] M. M. Kzkez, M. R. Barrault, and J. D. Craggs, "Spark channel formation,” J. Phys. D, Appl. Phys., vol. 3, no. 12, pp. 1886-1896, Dec. 1970.

[7] H. Koinuma, H. Ohkubo, and T. Hashimoto, "Development and application of a microbeam plasma generator," Appl. Phys. Lett., vol. 60, no. 7, pp. 816-817, Feb. 1992.

[8] E. Stoffels, A. J. Flikweert, W. W. Stoffels, and G. M. W. Kroesen, "Plasma needle: A non-destructive atmospheric plasma source for fine surface treatment of (bio)materials," Plasma Sources Sci. Technol., vol. 11, no. 4, pp. 383-388, Nov. 2002.

[9] E. Stoffels, I. E. Kieft, and R. E. J. Sladek, "Superficial treatment of mammalian cells using plasma needle," J. Phys. D, Appl. Phys., vol. 36, no. 23, pp. 2908-2913, Dec. 2003.

[10] E. A. Sosnin, E. Stoffels, M. V. Erofeev, I. E. Kieft, and S. E. Kunts, "The effects of UV irradiation and gas plasma treatment on living mammalian cells and bacteria: A comparative approach," IEEE Trans. Plasma Sci., vol. 32, no. 4, pp. 1544-1550, Aug. 2004.

[11] R. E. J. Sladek, E. Stoffels, R. Walraven, P. J. A. Tielbeek, and R. A. Koolhoven, "Plasma treatment of dental cavities: A feasibility study," IEEE Trans. Plasma Sci., vol. 32, no. 4, pp. 1540-1543, Aug. 2004. 
[12] I. E. Kieft, E. P. van der Laan, and E. Stoffels, "Electrical and optical characterization of the plasma needle," New J. Phys., vol. 6, no. 1, p. 149, Oct. 2004.

[13] I. E. Kieft, D. Darios, A. J. M. Roks, and E. Stoffels, "Plasma treatment of mammalian vascular cells: A quantitative description," IEEE Trans. Plasma Sci., vol. 33, no. 2, pp. 771-775, Apr. 2005.

[14] R. E. J. Sladek and E. Stoffels, "Deactivation of Escherichia coli by the plasma needle," J. Phys. D, Appl. Phys., vol. 38, no. 11, pp. 1716-1721, Jun. 2005.

[15] I. E. Kieft, J. J. B. N. Van Berkel, E. R. Kieft, and E. Stoffels, "Radicals of plasma needle detected with fluorescent probe," in Plasma Processes and Polymers, Plasma Processes and Polymers, R. d'Agostino, P. Favia, C. Oehr, and M. R. Wertheimer, Eds. Weinheim, Germany: Wiley VCH, 2005, pp. 295-308.

[16] M. Laroussi, "Sterilization of contaminated matter with an atmospheric pressure plasma," IEEE Trans. Plasma Sci., vol. 24, no. 3, pp. 1188-1191, Jun. 1996.

[17] H. W. Herrmann, I. Henins, J. Park, and G. S. Selwyn, "Decontamination of chemical and biological warfare (CBW) agents using an atmospheric pressure plasma jet (APPJ)," Phys. Plasmas, vol. 6, no. 5, pp. 2284-2289, May 1999.

[18] T. C. Montie, K. Kelly-Wintenberg, and J. R. Roth, "An overview of research using the one atmosphere uniform glow discharge plasma (OAUGDP) for sterilization of surfaces and materials," IEEE Trans. Plasma Sci., vol. 28, no. 1, pp. 41-50, Feb. 2000.

[19] M. Laroussi, "Nonthermal decontamination of biological media by atmospheric-pressure plasmas: Review, analysis, and prospects," IEEE Trans. Plasma Sci., vol. 30, no. 4, pp. 1409-1415, Aug. 2002.

[20] M. Vleugels, G. Shama, X. T. Deng, E. Greenacre, T. Brocklehurst, and M. G. Kong, "Atmospheric plasma inactivation of biofilm-forming bacteria for food safety control," IEEE Trans. Plasma Sci., vol. 33, no. 2, pp. 824-828, Apr. 2005.

[21] Y. Ma and R. E. Marquis, "Thermophysiology of Streptococcus Mutans and Related Lactic-Acid Bacteria," Antonie Van Leeuwenhoek, vol. 72, no. 2, pp. 91-100, Aug. 1997.

[22] E. A. Kidd, "Role of chlorhexidine in the management of dental caries," Int. Dent. J., vol. 41, no. 5, pp. 279-286, Oct. 1991.

[23] A. J. McBain, R. G. Bartolo, C. E. Catrenich, D. Charbonneau, R. G. Ledder, and P. Gilbert, "Effects of chlorhexidine gluconate-containing mouthwash on the vitality and antimicrobial susceptibility of in vitro oral bacterial ecosystems," Appl. Environm. Microbiol., vol. 69, no. 8, pp. 4770-4776, Aug. 2003.

[24] "Lasers in dentistry: Revolution of dental treatment in the new millennium," in International Congress Series 1248, I. Ishikawa, J. Frame, and A. Aoki, Eds. Amsterdam, The Netherlands: Elsevier, 2003.

[25] L. Zach and G. Cohen, "Pulp response to externally applied heat," Oral Surg., Oral Med., Oral Pathol., vol. 19, no. 4, pp. 515-530, Apr. 1965.

[26] J. Verhoef, "The phagocytic process and the role of complement in host defense," J. Chemother. Suppl., vol. 1, pp. 93-97, Jan. 1991.
[27] J. M. Gutteridge, G. J. Quinlan, and P. Kovacic, "Phagomimetic action of antimicrobial agents," Free Radic. Res., vol. 28, no. 1, pp. 1-14, Jan. 1998.

[28] A. N. Zaidel', V. K. Prokof'ev, S. M. Raiskii, V. A. Slavnyi, and E. Y. Shreider, Tables of Spectral Lines. New York: IFI/Plenum Data Co., 1970.

[29] R. W. B. Pearse and A. G. Gaydon, The Identification of Molecular Spectra, 3rd ed. New York: Wiley, 1963.

[30] W. J. M. Brok, M. D. Bowden, J. V. Dijk, J. J. A. M. Van der Mullen, and G. M. W. Kroesen, "Numerical description of discharge characteristics of the plasma needle," J. Appl. Phys., vol. 98, no. 1, pp. 013302-1-013302-8, Jul. 2005

J. Goree (M'92) received the Ph.D. degree from Princeton University, Princeton, NJ, in 1985.

$\mathrm{He}$ is currently with the Department of Physics and Astronomy, University of Iowa, Iowa City.

Bin Liu received the Ph.D. degree from the Institute of Physics, Chinese Academy of Sciences, Beijing, China, in 1999.

$\mathrm{He}$ is currently with the Department of Physics and Astronomy, University of Iowa, Iowa City.

David Drake received the Ph.D. degree from the University of Tennessee, Knoxville, in 1986.

He is currently with the Department of Endodontics, College of Dentistry, University of Iowa, Iowa City.

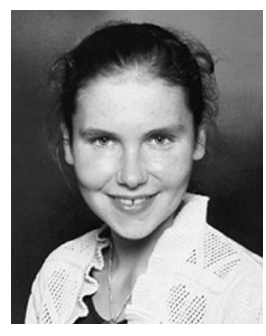

Eva Stoffels was born in October 1968 in Warsaw, Poland. She received the M.Sc. and Ph.D. degrees in plasma physics from Eindhoven University of Technology, Eindhoven, The Netherlands, in 1991 and 1994, respectively.

She is affiliated with Eindhoven University of Technology, where she leads research on nonthermal atmospheric plasmas and their biomedical applications. Her other research interests include low-pressure discharges for surface processing as well as electronegative and dusty plasmas. 